\title{
Stability of tillians and impact on the road network: case of the outside basin of Ouergha (Morocco)
}

\section{Stabilite des talus et impact sur le reseau routier: cas du bassin versant d'Ouergha (Maroc)}

\author{
I. JAOUDA ${ }^{1}$, A. AKHSSAS ${ }^{1}$, L. OUADIF ${ }^{1}$, L. BAHI ${ }^{1}$, A. LAHMILI ${ }^{1}$ \\ ${ }^{1}$ Mohammadia Engineering School, 3GIE Laboratory, Mohammed V University in Rabat, Morocco
}

\begin{abstract}
The study concerns the cartography of the spatio-temporal evolution of the embankments of the road network of the Oued Ouergha watershed using data from the Landsat images on the one hand, and the modeling of soil stability processes. On the other hand. This region experiences an intense destabilization linked to the combination of several natural factors, such as the brutality and the abundance of the precipitations and the outcrop on the marls and limestones of Cretaceous. Human intervention in this vulnerable environment accentuates its fragility through the clearing and degradation of vegetation cover. The analysis of satellite data has shown that the most degraded soils are not necessarily those with the highest rate of erosion over the past fifteen years and that some well-developed soils are unstable areas after clearing and their non-rational exploitation. Human intervention and the choice of road routes sometimes, combined with climate change, remain the most important factors in weakening and increasing the vulnerability of the slopes. The results obtained made it possible to identify and monitor vulnerable zones at the Ouergha watershed scale, where interventions are needed to limit the processes of slope degradation and their impact on the various road access routes in the region.
\end{abstract}

Key words: slope stability, road network, climate change, land use, Ouergha, Morocco.

Résumé. L'étude concerne la cartographie de l'évolution spatio-temporelle des talus du réseau routier du bassin versant d'Ouergha à partir des données des images Landsat d'une part, et la modélisation des processus da la stabilité des versants d'autre part. Cette région connait une déstabilisation intense liée à la conjugaison de plusieurs facteurs naturels, tels que la brutalité et l'abondance des précipitations et l'affleurement sur les marnes et calcaires du crétacé. L'intervention humaine dans ce milieu vulnérable accentue sa fragilité par le défrichement et la dégradation du couvert végétal. L'analyse des données satellitaires a permis de montrer, que les versants les plus dégradés ne sont pas forcément ceux qui ont le plus grand taux d'érosion au cours des quinze dernières années et que certains sols bien développés constituent des zones instables après défrichement et leur exploitation non rationnelle. L'intervention humaine et le choix parfois des tracés routiers, conjuguée avec les changements climatiques, restent les facteurs les plus importants dans la fragilisation et l'accentuation de la vulnérabilité des versants. Les résultats obtenus ont permis l'identification, le suivi des zones vulnérables à l'échelle du bassin versant d'Ouergha où des interventions sont nécessaires pour limiter les processus de la dégradation des versants et leur impact sur les différents accès routiers de la région.

Mots clés: Stabilité des versants, réseau routier, changements climatiques, occupation du sol, Ouergha, Maroc.

\section{Introduction}

Les marnes constituent plus de $70 \%$ des substrats qui affleurent dans le Pré-rif marocain et la majorité des sols fertiles de cette région se développent sur ce type de substrat. Ces sols constituent un capital important pour les habitants de cette. Les marnes sont des roches relativement tendres, elles subissent une géodynamique très active à leur surface et leur fragilité les rend très vulnérables aux aléas de la nature et à l'activité anthropique. Une combinaison de facteurs naturels et anthropiques a provoqué une érosion hydrique intense qui s'est manifesté par une dégradation des sols et des versants.
L'instabilité des versants dans le bassin versant de l'oued Ouergha est un phénomène complexe, tous les processus de dégradation s'y opèrent. La dégradation des versants est un processus qui se produit de façon inaperçue et qui est accéléré par l'activité anthropique. Elle dégrade le sol et affecte les la stabilité des versants et il a un impact direct sur les routes et les pistes.

Les routes et les pistes de la zone d'étude montrent différents types d'instabilité dues à la conjugaison de phénomène naturelles comme la pluviométrie importante essentiellement au niveau des zones qui peuvent être qualifiées de zone éclaires, et des caractéristiques lithologiques des formations géologiques de la région. Le diagnostique intégré et 
approfondi mené dans le bassin versant d'Ouergha a permis d'élaborer des documents cartographiques qui délimitent les zones vulnérables à l'instabilité des versants; pour orienter les priorités d'intervention et d'aménagement et aussi pour atténuer ou même éviter la dégradation des versant et protéger le milieu naturel.

\subsection{Présentation du périmètre d'étude}

Le bassin versant du barrage El WAHDA est drainé par l'Oued de l'Ouergha. Il s'étend sur une superficie de $6190 \mathrm{~km}^{2}$. Il se situe dans le Nord du Maroc entre les latitudes $34^{\circ} 25^{\prime}$ et $35^{\circ} 25^{\prime}$, et les longitudes $-5^{\circ} 24^{\prime}$ et $3^{\circ} 45^{\prime}$, il s'étend sur la majeure partie de la montagne rifaine.

Il est limité au Nord par la crête rifaine passant par Ketama, Bab Berred et Bab Taza, distant de la méditerranée d'environ $30 \mathrm{Km}$. Au Sud, par les bassins versants des affluents pré-rifains du Sebou et à l'Est par les affluents de la Moulouya et du Sebou du Périf oriental (Fig. 1).

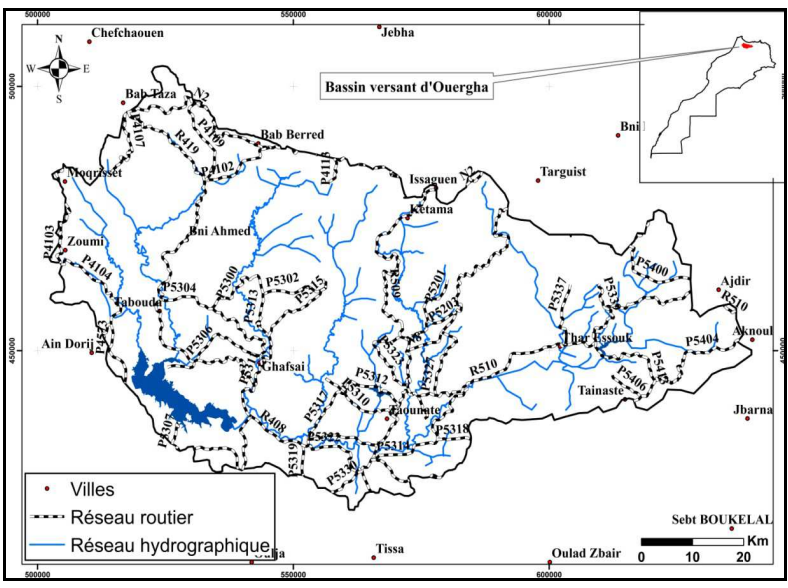

Fig. 1. Situation géographique et réseau routier du bassin versant du l'Ouergha.

Le bassin d'Ouergha est constitué en grande en grande partie par la nappe d'Aknoul et la nappe de Ketama (fig.2). Au nord affleurent, par endroits, des terrains géologiques appartenant aux zones méso-rifaine et prérifaine ([1]; [2]).

La nappe d'Aknoul constituent l'essentiel des affleurements surtout dans la moitié orientale du bassin, il s'agit surtout des marnes et calcaires du crétacé supérieur (Sénonien), auxquelles sont superposés localement des calcaires détritiques et marnes noires du Paléocène et de l'Eocène ([3]). Dans la moitié occidentale du bassin, s'étend le synclinal de Dhar Souk, occupé par des dépôts post-nappe marneux localement gréseux et conglomératiques du Tortonien et du Messinien.

Les formations du Jurassique et du Crétacé inférieur (grès et pélites, calcaires et dolomies) appartiennent à la nappe de Senhaja qui fait partie de la zone mésorifaine. Par contre, les marno-calcaires du Miocène moyen font partie de la zone pré-rifaine. L'unité de Ketama comporte un flysch schisto-gréseux du Crétacé inférieur épais de quelques Kilomètres ([4]).
L'unité de Tanger est considérée comme la couverture plus au moins décollée, de l'unité de Ketama, elle est formée par des schistes et des calcaires du Crétacé moyen et supérieur ([5]). La zone MésoRifaine apparaît au sud du bassin versant sous forme de fenêtre sous les nappes intra-rifaines. Les terrains de cette unité sont constitués par une série jurassicocrétacée, constituée dans la région de Taounate par des marnes surmontées par une formation molassique composée de grés et de conglomérats ([6]).

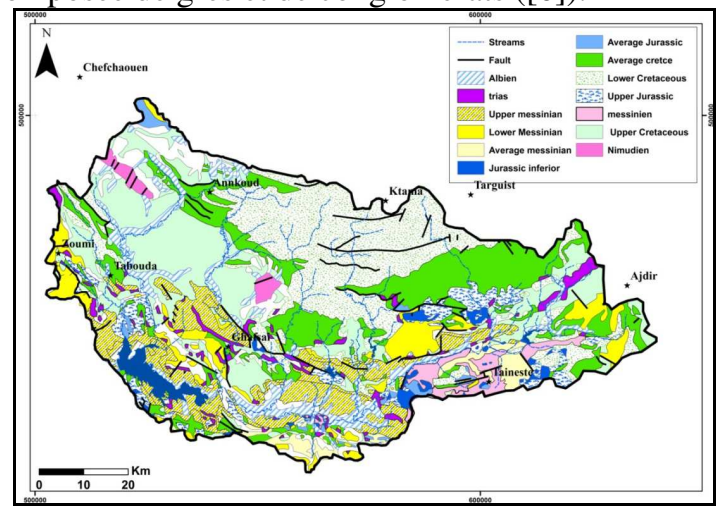

Fig. 2. Géologie du bassin versant du l’Ouergha.

Les lignes du relief sont fortement dépendantes des structures géologiques. Le relief est partout accidenté et accuse des pentes fortes à très fortes et n'est relativement atténué qu'en faveur des vallées. Le ruissellement a façonné des collines douces et arrondies sur les substrats marneux du Miocène supérieur et marneux ou marno-schisteux du crétacé supérieur ([7]).

Les lignes de crête les plus élevées ainsi que les hauts massifs sont façonnés sur les calcaires ou grès calcaires ou gréseux de l'Eocène et de l'Oligocène (fig. 3) du Miocène inférieur, et sur les calcaires du Jurassique. Ces hauts niveaux dominent des versants à pentes fortes. Par endroits, ils dominent aussi des versants à topographie ondulée témoignant de glissements successifs ayant sévis sur les versants marno-schisteux du Sénonien.

Dans les fonds des vallées des Oueds importants, notamment celle de l'Ouergha, se trouve des terrasses du Quaternaire récent et à topographie plane. Par endroits, sur les flancs de versants des vallées se rencontrent à des niveaux supérieurs aux précédents, des terrasses et replats datant du Quaternaire et d'époques plus anciennes ([7]). 


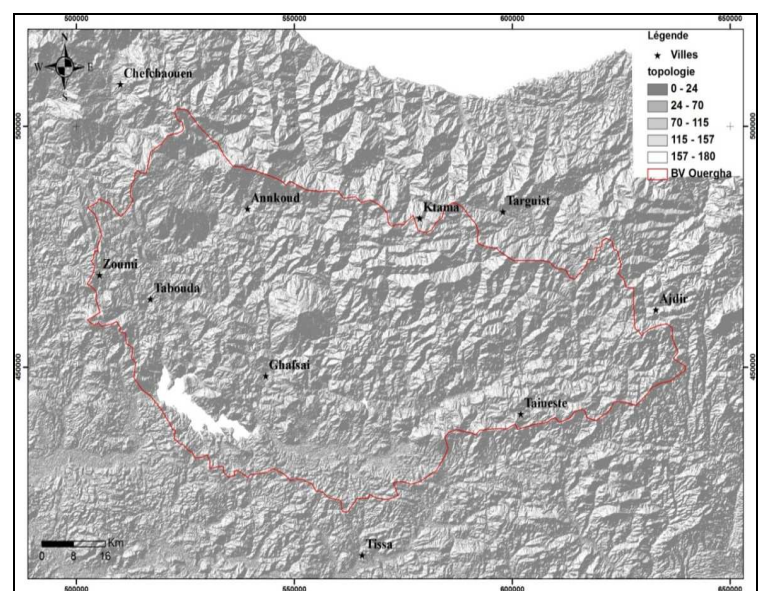

Fig. 3. Topographie du bassin versant de l'Ouergha.

En raison de son relief montagneux et sa situation géographique, la pluviométrie annuelle moyenne est de $650 \mathrm{~mm}$. Elle peut excéder les $1200 \mathrm{~mm}$ en année humide surtout sur les reliefs environnants situés à des altitudes plus élevées que celle d'Ouergha $(500 \mathrm{~m})$.

Les précipitations se répartissent principalement entre les mois d'octobre et mai. Décembre est le mois le plus pluvieux avec $87,5 \mathrm{~mm}$ ([8]). Les mois de juillet et août sont chauds et secs et les pluies ne dépassent pas $1,4 \mathrm{~mm}$ en moyenne.

L'agressivité des pluies, synthétisée à partir de la spatialisation des stations hydrologiques, montre que la valeur du facteur $\mathrm{R}$ varie de 66,7 à 128,7 (fig. 4).

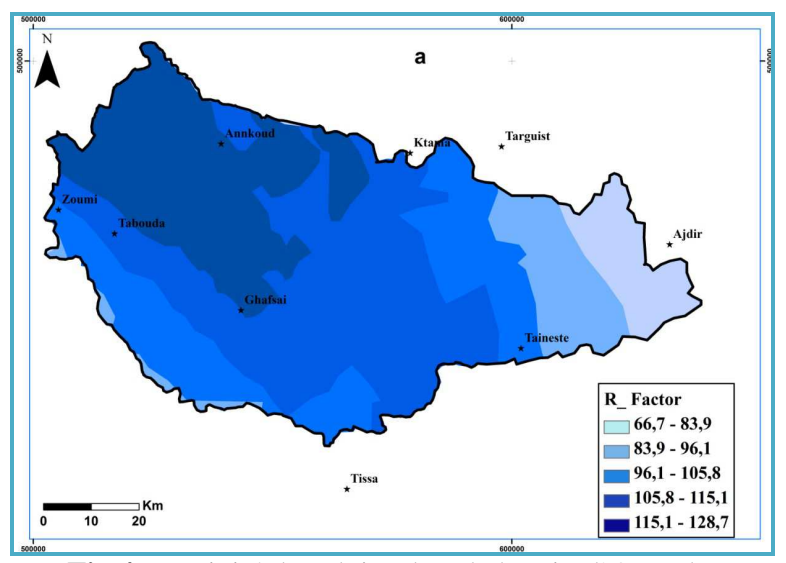

Fig.4. Erosivité des pluies dans le bassin d'Ouergha

La répartition géographique des différentes classes du facteur d'érosivité des pluies par rapport à la superficie totale du bassin est la suivante: $95 \%$ de la superficie du bassin est soumise à une érosivité de 70 à 119 et $5 \%$ de la surface du bassin versant est soumise à une faible érosivité.

Ces résultats sont proches de ceux obtenus par la DREF-NE ([9]) dans une étude réalisée sur le bassin versant d'Oued Beht limitrophe au bassin d'Ouergha ([10]).

Ainsi, il est rapporté, dans cette étude que l'agressivité pluviale est très concentrée dans l'intervalle 80 à 120 avec $86 \%$. La classe de forte érosivité dépassant 100 représente $24 \%$ du bassin versant d'Ouergha. Aussi, les valeurs obtenues sont élevées comparativement à celles du bassin versant de l'oued Boussouab dans le Rif oriental rapportées par Sadiki ([11]), qui ont trouvé que $\mathrm{R}$ varie de 31,2 à Anguied au Sud-Est du bassin à environ 60 au NordOuest et à celles du Rif central (bassin versant de l'oued Leben). Enfin, selon la classification de Marnique ([12]), le risque d'érosivité au niveau du bassin versant d'Ouergha est fort à très forte. Par conséquent, le facteur R pourrait entraîner l'apparition et le développement du phénomène de l'érosion linéaire.

Le réseau hydrographique du bassin versant Ouergha (Fig.5) s'écoule du NE vers le SW. L'oued Ouergha est le deuxième affluant principal de l'oued Sebou après l'oued Baht.

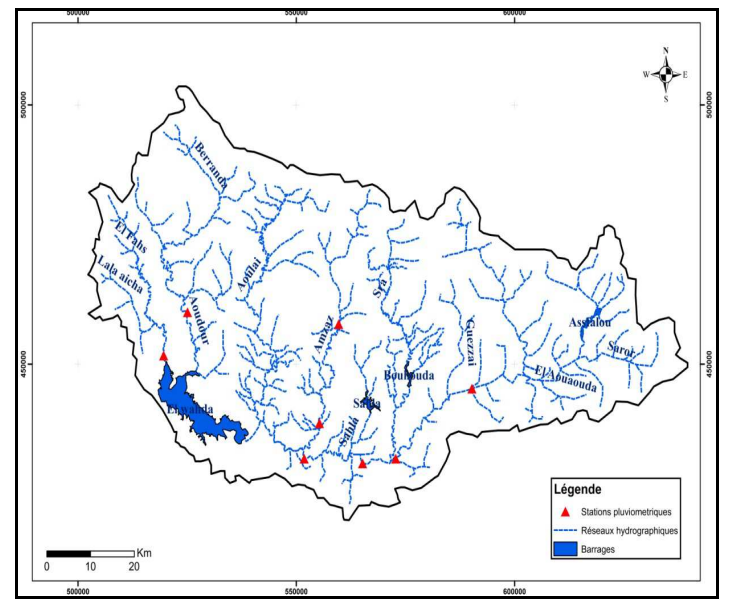

Fig.5: Carte hydrographique du bassin versant Ouergha.

L'oued Ouergha est forme sur la rive gauche par la réunion de l'oued Asfalou venant de l'Est, l'oued Teheris et l'oued Ras Ouergha venant du nord.

Il passe a la station hydrometrique de Bab-Ouender, en aval de laquelle l'oued Ouergha reçoit de gros affluents sur sa rive droite de l'aval versl'amont:

- L'oued Sra: contrôlé par la station hydrométrique de pont du Sker;

- L'oued Amzez: controlé par la station hydrométrique de Galez;

- L'oued Aoulai: contrôlé par la station hydrométrique de Rhafsai.

- L'oued Aoudour: contrôlé par la station hydrométrique de Tarfant ;

- L'oued Aoudai: peu avant de passe au site de barrage Al Wahda et a la station hydrométrique de M'Jara, immédiatement a l'aval du site.

Le bassin versant Ouergha renferme quatre barrages, de l'amont vers l'aval: Asfalou, Bouhouda, Sahla el Al-Wahda, ce dernier est le plus grand aménagement hydraulique du Maroc et le deuxième en Afrique.

De point de vue pente, sept classes ont été identifiées. La classe 6-16\%, éparpillée sur tout le 
territoire, représente $43 \%$ de la surface du bassin versant (fig. 6).

La classe 15-25 est localisée surtout dans l'amont et au centre du bassin versant sur $23 \%$ de la superficie du bassin versant.

Les pentes dépassant $25 \%$ sont localisées dans l'amont sur une superficie de $18,5 \%$ du bassin versant. Ces résultats montrent le caractère accidenté du bassin versant de l'Ouergha.

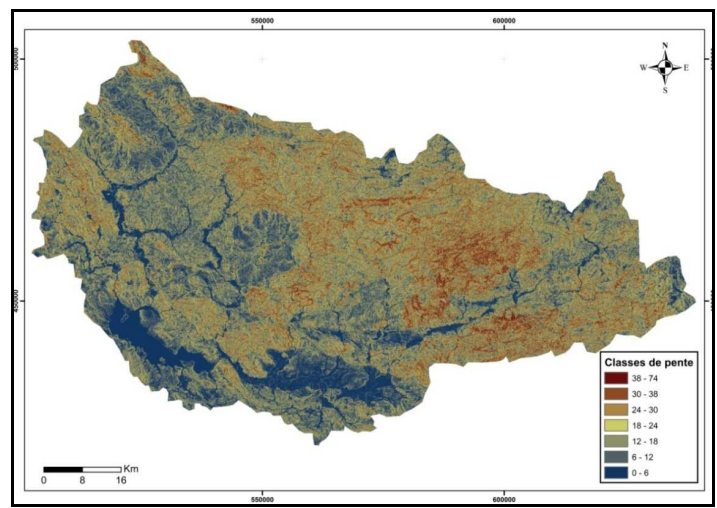

Fig.6. Carte des pentes du bassin versant de l'oued Ouergha.

L'occupation actuelle des terres: La carte présentant les classes d'occupation du sol du périmètre étudié (fig. 7) a été obtenue suite aux résultats de traitement des images satellitaires et de classification des bondes.

En effet, six principaux types d'occupation du sol (forêt, terrains fortement dégradées ...).répartis sur l'ensemble de la zone d'étude.

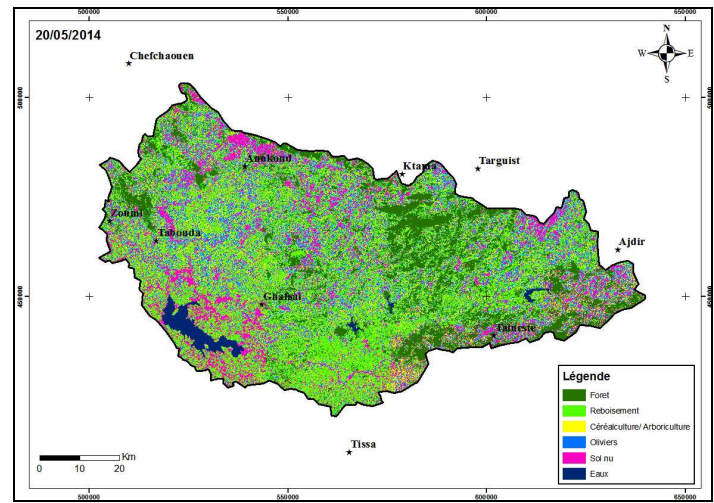

Fig.7. Carte de l'occupation des sols du bassin versant Ouergha.

L'érosion comme par ailleurs dans le Rif, la dégradation des versants au niveau du périmètre est importante et y constitue une contrainte majeure (fig. 8).

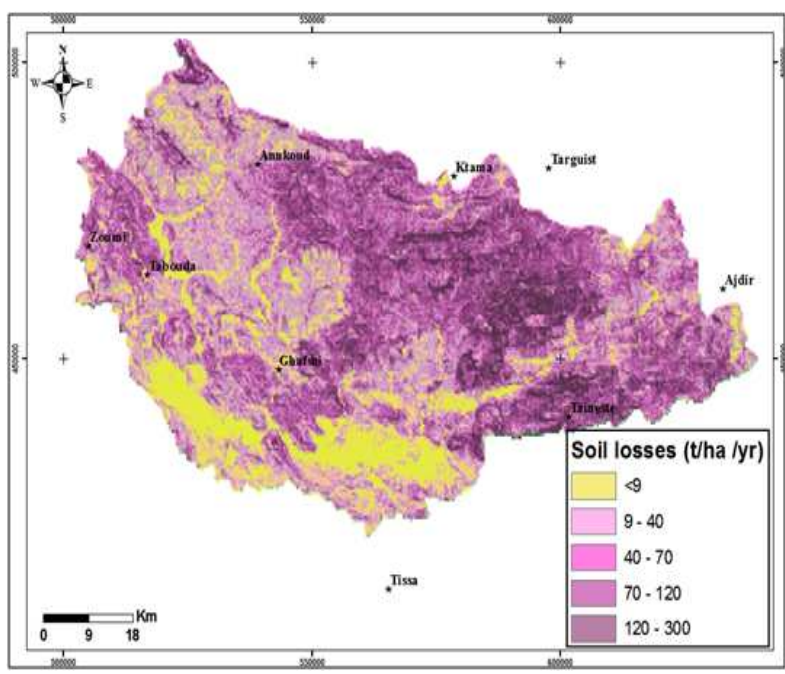

Fig.8. Carte des pertes en sols dans l'Ouergha.

Elle s'y manifeste par des formes et intensités variables. Et cela pour des raisons lithologiques et structurales, climatiques et anthropiques.

La carte géologique (Fig. 2) montre l'extension importante des substrats tendres de différents âges et qui sont sensibles à la dégradation telles que les marnes, les pélites, les marno-schistes et dans une certaine mesure les marno-calcaires.

Les pentes moyennes à fortes favorisent les processus d'érosion. En effet, les pentes dépassant $20 \%$ totalisent $50 \%$ de l'ensemble des classes de pentes. La couverture végétale peu dense, protège mal les versants contre le ruissellement provoqué par des pluies agressives. Enfin, le surpâturage et l'usage inadéquat des terres sont aussi des facteurs qui favorisent la dégradation des terres.

\section{Méthodologie}

Toutes les cartes (fig. 9) ont été saisies, traitées et analysées à l'aide de l'outil SIG, notamment le logiciel Arc-gis.

La combinaison des ces couches thématiques à l'aide de l'outil précité a abouti à la réalisation de la carte de stabilité des versants.

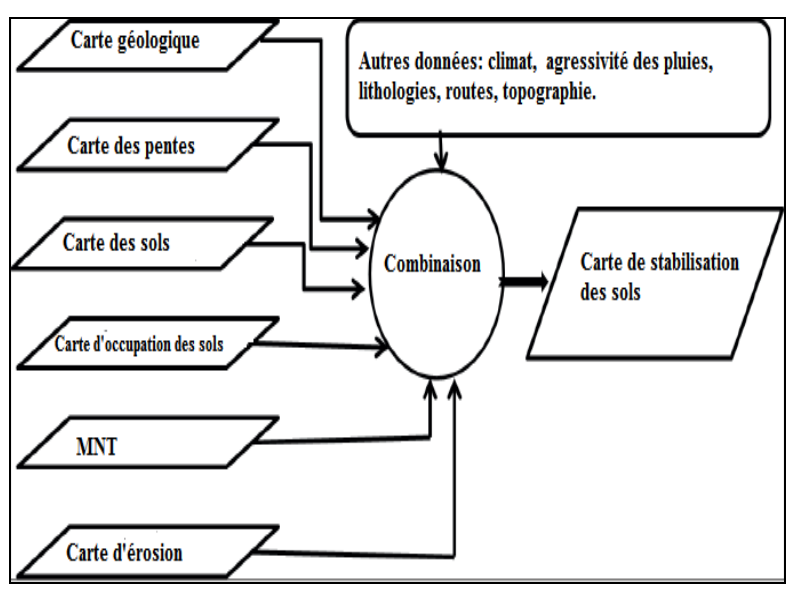

Fig. 9. Combinaison des différentes cartes thématiques à l'aide du SIG. 


\section{Résultats et discutions}

Du fait qu'elles sont toutes géo-référencées dans le même système de coordonnées, leur superposition et confrontation ont permis donc la délimitation des unités cartographiques de la carte résultante précitée. Cette dernière est obtenue suite à la combinaison thématique de toutes ces couches analysées en mode raster.

En effet, une fois la superposition réalisée, les valeurs des pixels homologues dans chacune des couches sont analysés et regroupés dans des classes aussi homogènes que possible répondant en fonction de leur caractéristiques à des besoins spécifiques en matière de gestion et de stabilisation.

Ces classes correspondent donc aux différentes unités cartographiques de la carte de figure 9. Une méthodologie similaire été adoptée dans l'étude et l'évaluation des terres d'un versant situé dans la plaine de Tadla ([14]). Les unités de la carte de la figure 10 correspondant donc à des catégories de terre dont les recommandations tiennent compte de la variabilité de leurs caractéristiques spatiales locales présentées dans les cartes ci-dessous cartes géologique, lithologie, pédologique, relief et occupation du sol et routes.

L'analyse des facteurs du milieu, surtout le climat et les sols fait ressortir que les principales contraintes des ces derniers sont la pente et la dégradation des versants.

En plus des facteurs précités, les recommandations prennent aussi en considération l'aptitude des terres ([15]; [16]).

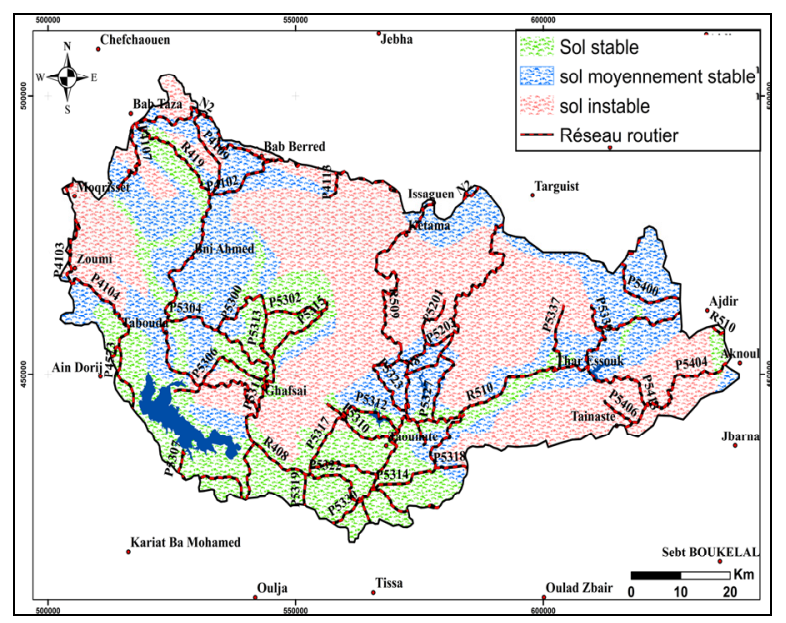

Fig. 10. Carte de stabilité des versants de l'Ouergha.

La carte de stabilité des versants reforme trois types des versants:

Versant stable: Les terrains délimités dans cette catégorie concernent une grande partie du périmètre. Ils s'étendent au nord et à l'ouest de la ville de Dhar souk sur les dépôts du Miocène post-nappe et sur les marnoschistes de la nappe d'Aknoul. Les pentes sont inférieures à $20 \%$ et le relief dans l'ensemble est collinaire. Les sols sont essentiellement des sols peu évolués d'apport colluviaux sur colluvions de marnes miocène et marno-schistes du crétacé supérieur et secondairement des marnes noires de l'Eocène et du Crétacé inférieur.

Cette catégorie de terre englobe aussi et d'une manière très localisée, les sols alluviaux irrigués des basses terrasses quaternaire, à relief plat, occupant le fond des vallées des principaux oueds, notamment de Oued Ouergha, Illustrant la situation inquiétante dans la zone 1 tout en impactant les routes (Photo1, Fig. 13).

Versant moyennement stable: Les pentes des versants varient de 20 à $35 \%$. Les terrains sont surtout des marno-calcaires du Miocène moyen, des marnes de l'Eocène moyen et parfois des calcaires et dolomies du Lias inférieur. Les sols dominants sont des sols d'érosion régosoliques. Les causes principales de l'instabilité des versants sont la topographie, l'érosion hydrique et la pente (Photo 2, Fig. 12).

Versant instable: Les pentes de cette catégorie de terre sont fortes et dépassent 35. L'altitude est plus importante en moyenne, elle est de $1200 \mathrm{~m}$. Les terrains sont caractérisés par les marnes du Miocène supérieur, et grès et pélites du Jurassique supérieur. Les sols sont surtout des sols peu évolués.

L'érosion est active par ravinement et donne parfois des badlands, ce phénomène provoque l'instabilité des versants, la fragilité des tracés routiers et la perturbation des routes dans les cas extrêmes (Photo 2, Fig.12).

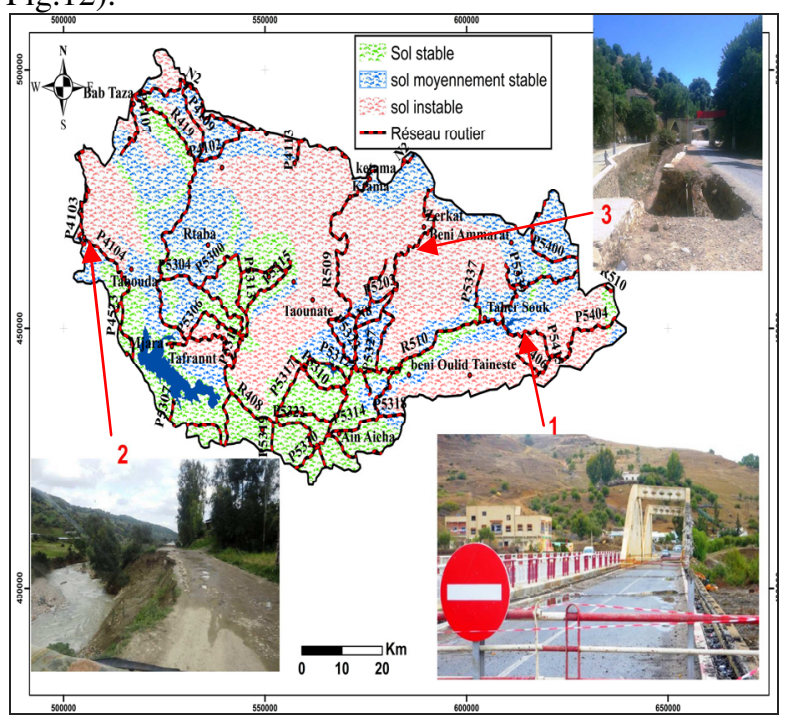

Fig. 12. Carte de stabilité des versants; Photo1 Pont Ain Aaicha sur Oued Ouergha (affaissement); photo2: Ain Aicha S302 (tassement); Photo 3: Zoumi P4103 (glissement).

\section{Conclusion}

L'utilisation de l'imagerie satellitaire et du SIG a permis de délimiter les zones où les versants constituent un danger sur les tracés routiers inventoriés et sur les pistes qui relient les différentes agglomérations de la zone d'étude. Les versants instables se situent dans la partie centrale du bassin (couleur rouge fig.10), les zones moyennement stables 
se trouve dans la partie Est du bassin versant et aussi sur la partie Ouest de bassin.

Cette instabilité dépend essentiellement de La nature des terrains qui sont constitués des marnes et calcaires du crétacé supérieur, auxquelles sont superposés localement des calcaires détritiques et marnes noires du Paléocène. La forte pente qui dépasse les $35 \%$. La pluviométrie importante, elle peut excéder les $1200 \mathrm{~mm}$ en année humide surtout sur les reliefs environnants situés à des altitudes plus élevées que celle d'Ouergha $(500 \mathrm{~m})$ et la lithologie des sols en plus de la dégradation des couvert végétal qui protège les versants conte l'instabilité et rendre les zones stable naturellement.

La réalisation de la carte de stabilité des versant découle l'analyse et l'intégration des différentes caractéristiques morpho-géo-pédologiques influençant la stabilité des terres et constituant autant de thèmes organisés dans la base de données spatiales. Cette dernière peut être actualisée et alimentée au besoin par d'autres informations complémentaires. Elle peut ainsi aider les responsables du développement de la zone dans l'orientation et la prise de décisions en matière d'utilisation rationnelle et de conservation des versants soumis à des processus d'instabilité très importants.

\section{Bibliographie}

1. J.C Vidal. Carte géologique du Rif: Taïneste Echelle 1/50 000, échelle 1/50 000 Direction de la géologie. Ministère de l'Énergie et des Mines. Royaume du Maroc (1983).

2. Leblanc D., Feinberg H. coll., Lorenz H.GCarte géologique du Rif: Taïneste, échelle 1/50000. Direction de la géologie. Ministère de l'Énergie et des Mines. Royaume du Maroc (1983).

3. Asebriy L., Bourgois J., Cherkaoui T.E, Azsimousa A. Evolution tectonique récente de la zone de faille du Nékor: importance paléogéographique et structurale dans le Rif externe, Maroc. Journal of African Earth Sciences (and the Middle East), volume 17, Issue 1, July Pages 65-74 (1993).

4. Asebriy L. Bourgois J. Leiking M. Sur l'âge du métamorphisme anchi-épizonal de l'unité de Ketama, Rif central (Maroc). C. R. Acad. Sci. Paris, t. 313, Série II, p. 787-793 (1991).

5. Asebriy L., Azdimousal A., Jabaloy A., Booth-Rea G., González-Lodeiro F. and Bourgois J. Lithostratigraphy and structure of the temsamane unit (eastern external rif, morocco). Revista de la Sociedad Geológica d'España, 20 (3-4) (2007).

6. Leblanc D. Etude géologique du Rif externe oriental au Nord de Taza (Maroc). Notes et Mémoires, Service Géologique Marocain, Rabat, 281: 1-159 (1979).

7. Asebriy L., Azdimousa A., Bourgois J., Poupeau G. \& Montigny R. Histoire thermique et surrection du Rif externe et des nappes de flyschs associées (Nord
Maroc). Trav. Inst. Sci., Rabat, sér. Géologie \& Géogr. phys., 21, 15-26 (2003).

8. Ihissou Y. Évaluation de l'impact de l'arboriculture fruitière en zones de montagnes sur la conservation des sols et des eaux: cas de la zone de Tahar-souk (Province de Taounate). PFE, ENA Meknès, 95p (2014).

9. DREF du Nord-Est: Etude d'aménagement du bassin versant d'Oued Beht en amont du barrage El Kansera, Direction Régionale des Eaux et Forêts, Fès (2007).

10. El Gaatib R., Larabi A. et Mohamed Faouzi1 M. Integrated elaboration of priority planning of vulnerable areas to soil erosion hazard using Remote Sensing and GIS techniques: A pilot case of the Oued Beht Watershed (Morocco), J. Mater. Environ. Sci. 6, 11, 3110-3127, ISSN: 2028-2508 CODEN: JMESCN (2015).

11. Sadiki A, S. Bouhlassa, J. Auajjar, A. Faleh and J. Macaire, Utilisation d'un SIG pour l'évaluation et la cartographie des risques d'érosion par l'Equation universelle des pertes en sol dans le Rif oriental (Maroc) : cas du bassin versant de l'oued Boussouab, Bulletin de l'Institut Scientifique, Rabat, section Sciences de la Terre, 26, 69-79, (2004).

12. Manrique L.A. Land Erodibility assessment methodology, LEAM, Using soil survey data based on soil taxonomy, University of Hawaii, Editorial and publication Shop, Honolulu, Hawaii USA, 28p (1988).

13. Ministère de l'Agriculture et de la Mise en valeur Agricole. Étude de reconnaissance au 1/100 000 en vue de la mise en valeur agricole dans les cercles de Taounate et Ghafsai, Province de Taounate (1994).

14. Loukili M., Bock L., Engels P., Mathieu L. Approche géomorpho-pédologique et Système d'Information Géographique (SIG) pour la gestion des terres au Maroc. EGS 7: 37- 52 (2000).

15. FAO (1976). Cadre pour l'évaluation des terres. Bulletin pédologique de la FAO, 32, Rome, 64 p.

16. FAO Guidelines for land evaluation for rainfed aqriculture. FAO soils bulletin, 52, Rome, 237p (1983). 17. Logiciel Google earth

18. (https://www.google.com/intl/fr/earth/). 